\title{
INFORTUNIO DE LA INVESTIGACIÓN SOCIAL CUALITATIVA EN LA ERA DEL CAPITALISMO ACADÉMICO
}

\author{
Begoña Abad Miguélez ${ }^{1}$ y Andrés Davila Legerén ${ }^{2}$ \\ ${ }^{1 y 2}$ Universidad del País Vasco/Euskal Herriko Unibertsitatea, España \\ ${ }^{1}$ begona.abad@ehu.eus; ${ }^{2}$ andres.davila@ehu.eus
}

\begin{abstract}
Resumen. Es notable el interés que la investigación cualitativa demuestra tanto por el estudio del uso y organización del tiempo como por la construcción social de categorías temporales. En cambio, no lo es tanto el interés acerca del papel que la dimensión temporal juega en la conformación de la propia investigación cualitativa. Al tomar en consideración tal dimensión percibimos que esta investigación requiere darle tiempo al tiempo, demorándose en su propio proceso de desarrollo; lo cual choca frontalmente con la crono-lógica imperante en el capitalismo académico actual dando pie al infortunio epistemológico de la investigación social cualitativa en tanto que investigación lenta. En este artículo se revisa brevemente el alcance de dicho infortunio: primero, prestando atención al contexto en el que se enmarca; describiendo después los momentos constitutivos de la investigación social cualitativa; concluyendo con una reflexión de futuro como propuesta hacia la afirmación de la lógica epistemológica de la investigación cualitativa.
\end{abstract}

Palabras clave: Investigación Cualitativa; Investigación Lenta; Modos de Producción de Conocimiento; Lógica Temporal en la Investigación; Capitalismo Académico.

\section{MISFORTUNE OF QUALITATIVE SOCIAL RESEARCH IN THE TIMES OF ACADEMIC CAPITALISM}

\begin{abstract}
The interest qualitative research shows is remarkable both for the study of the use and organization of time as well as for the social construction of temporal categories. The focus is not so much on the role that the temporal dimension plays in the formation of qualitative research itself. By taking into account such a dimension we perceive that this research requires letting time take its course, taking time in its own development process. This logic collides head-on with the chronologic prevailing in today's academic capitalism, giving rise to the epistemological misfortune of qualitative social research as a slow research. This article briefly reviews the scope of this misfortune. We start by paying attention to the context in which it is framed; then we will continue with the description of the constituent moments of qualitative social research in order to conclude with a proposal for the future: the necessary reaffirmation of the epistemological logic of qualitative social research.
\end{abstract}

Keywords: Qualitative Research; Slow Research; Modes of Knowledge Production; Temporal Logic in Research; Academic Capitalism.

\section{INTRODUCCIÓN}

Múltiples y diversas son las investigaciones cualitativas que, tanto desde la sociología como desde la antropología, se han venido realizando en torno al uso y organización del tiempo, pero también sobre la construcción social de categorías temporales diferenciando, en este caso, tanto tiempos como temporalidades.

Sin embargo, a menudo se desdeña la intervención y alcance de la propia dimensión temporal en las hechuras de una investigación social cualitativa. 
Al tomar en consideración tal dimensión se percibe que la realización de este tipo de investigación requiere darle tiempo al tiempo, para producirse incluso a contratiempo, a tenor de la crítica sobre sí misma, y sobre la simultaneidad y circularidad de sus momentos y tareas, que tiñe de reflexividad su propio proceso de realización.

De este modo, vista desde la lógica temporal que la atraviesa, la investigación social cualitativa se nos revela como una investigación lenta (slow science) que encuentra en la demora y la desaceleración necesarias para escuchar lo que la realidad en estudio tiene que decir su razón de ser, tanto ética como epistemológicamente. Desafortunadamente, esta característica en la que se basa su valor epistémico es también la fuente de su infortunio en el momento actual, o mejor aún, bajo la égida de lo que se ha venido conociendo como capitalismo académico, una expresión más de la hegemónica razón neoliberal. Conviene recordar aquí que la sujeción a la lógica mercantil que define esta forma de capitalismo da lugar a una ciencia, así como a una investigación, caracterizada por: la eficiencia (orientación a outputs directa o inmediatamente aplicables); la calculabilidad (utilización de criterios controlables y cuantitativos, en ocasiones externos a las exigencias de la propia disciplina, para evaluar la calidad de la investigación); y la predictibilidad (articulación anticipatoria de resultados previstos e impacto). Da lugar también a una crono-lógica que rige los proyectos de investigación tanto como las carreras (y no sólo en el sentido goffmaniano) desde el criterio de la aceleración o investigación rápida (fast science): producir resultados de impacto en el menor tiempo posible.

En este contexto, el infortunio de la investigación social cualitativa se asocia a la necesidad de ajustarse a la lógica temporal imperante lo que se traduce, en última instancia, en la traición a las bases epistemológicas que la sustentan porque, al fin, la lógica temporal de la investigación es parte sustancial de su lógica epistemológica. Así, el objetivo de este artículo es profundizar en el alcance del infortunio epistemológico de la investigación social cualitativa tal y como se presenta y experimenta en el momento actual.

\section{RÉGIMEN DE PRODUCCIÓN DE CONOCIMIENTO Y CAPITALISMO ACADÉMICO}


En el actual modelo económico, la producción, comercialización y transferencia intensiva de conocimiento entre el mundo académico y los mercados se percibe como factor clave para el logro económico y la competitividad en el seno de lo que se ha venido conociendo como capitalismo académico, concepto acuñado por Slaughter y Leslie (1997).

La seña de identidad de esta forma específica de capitalismo es la sujeción del mundo académico de la educación superior, y la investigación a él asociada, a la lógica mercantil. Así, la educación superior se mercantiliza hasta convertirse y funcionar como una empresa de producción de conocimiento y cualificaciones tanto válidas como validadas en términos económicos y mercantiles (Cannizzo, 2015, p. 200) siempre en liza o competencia con otras empresas similares por el logro de recursos de financiación procedentes del sector privado, es decir, del propio mercado, una vez retirado el Estado de la gestión y control de los servicios básicos. Este viraje hacia la entrepreneurial university afecta a docentes y discentes. El alumnado se convierte en cliente dispuesto a recibir un producto empaquetado y de calidad susceptible de ser rentabilizado a corto plazo en un futuro laboral de éxito. El profesorado, por su parte, y en tanto que proveedor del servicio dentro de la marca-empresa a la que pertenece, se somete a una dinámica en la que se verá en la obligación de mantener un crecientemente alto ritmo de producción (enseñar, investigar, publicar, auditar, calificar, recabar financiación...) si desea mantener su posición dentro de la empresauniversidad. Convertidos en empresarios o emprendedores de sí mismos, se espera de ellos que adopten como valores esenciales la flexibilidad y la capacidad de adaptación, la destreza para venderse y la habilidad para competir en la gestión de un tiempo propio dominado por el logro y el complimiento de proyectos-productos. Así, "research work is project work, involving writing project applications, finding project partners, competing for project funding, recruiting project researchers, running project management, meeting project deadlines and reaching the goals defined in the project contract" (Ylijoki, 2016, p. 11).

Podemos hablar de un proceso de proletarización en el contexto de la entrepreneurial university (Gallas, 2018), similar al sufrido por el resto de trabajadores inmersos en la lógica del capitalismo global. El primer síntoma/indicador de esta proletarización es la pérdida de autoridad de la academia en favor de un profesionalismo técnico (Schwant, 2008; citado en Koro-Ljungberg \& Barko, 2012, p. 257) a tenor del cual los profesionales se transmutan en proveedores de servicios expertos, o simples "mediadores" (Mills \& Ratcliffe, 2012, p. 6) en un mercado que, lejos de sustentar sus criterios de calidad y status en valores puramente intelectuales, se rige por indicadores de productividad e impacto; y que, lejos de poner en 
valor la identidad del homo academicus (Bourdieu, 1992), ensalza la figura del academic entrepreneur sometido al terror de la performatividad (Ball, 2003; Morrish \& Sauntson, 2016; Vostal, 2015), esto es, al juicio permanente de su contribución a la cadena de valor, a la productividad.

La proletarización se acompaña también de precarización vivida y sentida como estrés, culpa y angustia (Ryan-Flood \& Gill, 2010). Y no debemos olvidar que el alcance de esta precarización vital se hace presente en el modelo propuesto de universidad/academia, y su gobierno tácito del tiempo de docencia e investigación, en la delimitación de la frontera dentro/fuera del sistema instituyendo la distinción entre quién puede estar dentro por ser capaz de coordinar su tiempo con el tiempo del sistema (logrando oportunidades, poder, recursos y reconocimiento), y a quién se excluye (relegándosele a la interrupción y ralentización de su carrera) por ser incapaz de gestionar esa sincronización.

Como colofón, el capitalismo académico también delimita, en el interior del sistema, un modo de producción de conocimiento (qué se puede hacer, qué se puede conocer o qué conocimiento se puede producir) de modo que la investigación orientada al conocimiento queda ahora orientada a responder, tanto temática como metodológicamente, a los intereses del mercado de la forma más rápida (obteniendo la mayor cantidad de información en el menor tiempo posible) y efectiva (obteniendo la mayor cantidad de datos veraces, contundentes, cuantificables y traducibles a soluciones inmediatamente aplicables). Dicho de otro modo, el capitalismo académico sólo deja lugar para una ciencia/investigación rápida, aplicada, dirigida a la obtención y distribución de outputs directamente orientados a resolver problemas concretos (efficiency); una ciencia/investigación que utiliza criterios controlables y cuantitativos (aunque externos a las exigencias de la propia disciplina) para evaluar la calidad de la investigación a partir de indicadores de impacto, número de publicaciones asociadas al proyecto de investigación, etc. (calculability); y, finalmente, una ciencia/investigación basada en la anticipación del futuro (predictability) a partir del conocimiento presente (Adams et al. 2009), potencial que se manifiesta ya en el propio proyecto-propuesta de investigación a través de la articulación tanto de los resultados previstos como del impacto esperado.

Todo esto da lugar a una producción intensiva de conocimiento que encaja en los parámetros del modo de producción que Michael Gibbons et al. (1994) describen como colaborativo y transdisciplinar en oposición al modelo tradicional/jerárquico y disciplinar. Más 
allá de la posible simplificación descriptiva que puede entenderse en este trabajo original, lo interesante para nosotros, siguiendo a Simon Smith (2015, p. 152-153), es la perspectiva temporal subyacente a cada uno de los modelos propuestos. Utilizando la nomenclatura de Gibbons y sus colaboradores, el modo 1 (tradicional y disciplinar en tanto en cuanto se organiza en torno a las exigencias de la disciplina) se estructura en torno a una temporalidad larga ( $y$ en cierto modo lenta), mientras que el modo 2 (transdisciplinar y colaborativo) se estructura en torno a una temporalidad rápida condicionada por aquellas exigencias y necesidades propias de cada proyecto/problema planteado para ser resuelto en el cortomedio plazo.

Lo que diversas fuentes (Ylijoki 2016; Vostal, 2015) denominan project-time (o tiempoproyecto), se convierte así en la lógica temporal que determina la investigación en el capitalismo académico. Dicha concepción del tiempo comporta una lógica de investigación en la que el marco temporal es fijo y estricto, al estar marcado por la realización secuencial de actividades predefinidas de forma que el tiempo global del proyecto, y el específico para cada una de esas actividades, puede ser medido y cuantificado. Implica, igualmente, una lógica lineal, acumulativa y progresiva pues "guided by schedules, research moves forwards steadily towards the goals that have been defined in the contract. Each new phase is based on the previous phase so that the results are produced in accumulative chain" (Ylijoki, 2016, p. 14). Por la misma razón, y dado que "it is based on dates, timings, durations and sequences which can be quantified, measured and evaluated by the clock and calendar without taking into the account the particular work conditions under which research is carried out" (Ylijoki, 2016, p. 15), resulta que es invariante e independiente del contexto de producción.

La trascendencia del tiempo proyecto como eje estructurador de la lógica de investigación va más allá de la simple construcción de una tipología. Con el predominio del tiempo proyecto, y la fast science con que es asociado, se produce la transmutación del principio legitimador del modo de producción del conocimiento (epistemología) y del modo de entender la realidad (ontología). Cabe recordar, en este sentido, que buena parte de la tradición epistemológica occidental se ha sustentado sobre metáforas espaciales (horizontes, campos, bordes, esferas, territorios, etc.) asociadas, de un modo u otro, a una gramática de poder, dominación y control (Pascale, 2011) que viene siendo ejercida a través de la configuración de listas, categorizaciones y mapas entendidos, todos ellos, como dispositivos de metaforización espacial de la producción de conocimiento. Estos dispositivos 
separan el espacio del tiempo dando prevalencia al primero lo cual explicaría por qué en la legitimación de la epistemología occidental tradicional y dominante la lógica temporal ha venido siendo excluida del razonamiento en favor del uso de tropos espaciales (Pels, 2003). Sin embargo, en el contexto actual (donde el lema "the faster, the better" se convierte en credo), quizás haya llegado el momento de replantearse el argumentario tropo-espacial para incluir en él al tiempo como eje estructurador. $\mathrm{Y}$ no sólo porque para obtener una comprensión holística de la realidad estudiada (el qué de la indagación) sea necesario atender simultáneamente tanto al eje espacial (el dónde de la indagación) como al temporal (el cuándo), sino porque pensar la investigación (la investigación social en el caso que nos ocupa) desde el eje temporal nos lleva a plantearnos las posibilidades pero, sobre todo, los límites, desventuras e infortunios de una investigación "lenta" en un contexto de aceleración generalizada como el propiciado por el capitalismo académico que hemos venido describiendo hasta aquí.

\section{DESVENTURAS TEMPORALES DE LA INVESTIGACIÓN LENTA A TENOR DEL CAPITALISMO ACADÉMICO}

Hace ya unos años que se publicó la biografía de la genetista Barbara McClintock, la descubridora de los llamados "genes saltarines" o "transposones" (Keller, 1983). En este trabajo se afirmaba que su éxito radicaba en su paciencia, en su capacidad para tomarse el tiempo necesario para observar y escuchar lo que la materia con la que trabajaba tenía que decir y mostrar (Levy, 2007). Es evidente que esta mujer, ganadora del Premio Nobel de Medicina en 1983, entendía la ciencia como investigación lenta, pausada, que se toma su tiempo para pensar, planificar, diseñar, experimentar, observar, entender, concluir, etc. Es decir, un modo de hacer ciencia que lejos de recrearse en el timing, visto como tiempo lineal centrado en la búsqueda de lo que ya sabemos o sospechamos, se centra en la dureé (cf. Henri Bergson, por ejemplo en Deleuze, 2017), contemplándose en esta definición del tiempo los momentos de descubrimiento en que encontramos aquello que no sabíamos que buscábamos.

La lógica temporal de la investigación lenta por la que aboga Barbara McClintock, si bien de una manera intuitiva o no intencionada, y otros autores, esta vez de una manera abiertamente manifiesta y política (O'Neill, Martell, Mendick \& Müller, 2014) se ajusta a lo que Oilin-Helena Ylijoki (2016) denomina process-time o tiempo-proceso. Frente al modelo dominante de la fast science, cuyo régimen temporal se engloba dentro de lo que hemos descrito anteriormente como tiempo-proyecto, el régimen temporal de la slow science, o 
tiempo-proceso, no conlleva límites temporales estrictos lo que da lugar a fluidez y movimiento (con continuidades y rupturas, etc.); no se ajusta a la dictadura de un calendario/cronograma lineal sino que sigue los dictados de su tempo interno (aquel marcado por el progreso creativo de cada actividad emprendida) sea cual sea su conformación (lineal, cíclico, pendular, etc.) dando lugar a momentos tanto de avance, como de vuelta al inicio, tanto de movimiento como de espera, tanto de aceleración como de desaceleración, momentos que adquieren sentido en y desde el contexto de investigación en el que se insertan, motivo por el cual el tiempo proceso es impredecible, encarado hacia un futuro abierto en su potencial espacio de emergencia (Ylijoki, 2016, p. 15). De hecho, en este esquema temporal carece de sentido cerrar ese potencial futuro definiendo los resultados esperados a priori, antes incluso de realizar la investigación, porque la investigación entendida desde el tiempo-proceso produce resultados inesperados, preguntas que ni siquiera nos habíamos planteado al inicio del proceso. Preguntas que no podríamos plantear en ese momento inicial porque la base de la indagación en esta investigación lenta no se sustenta en las normas de probabilidad, regularidad, frecuencia, simetría, etc. vistas como sinónimo de certeza científica rápidamente verificable (Torrance, 2008). La base de esta indagación se halla en lo que Maggie MacLure (2013) denomina the wonder of data, esto es, en la atención prestada a lo imprevisto (un comentario en una entrevista), a lo tangencial (una anécdota que se cuela en el discurso), a lo inesperado (una expresión facial involuntaria y significativa), en definitiva, a la capacidad performativa de la realidad (Denzin, 2013, pp. 354-355).

Y aquí se plantea una paradoja relevante pues si bien, tal y como nos recuerdan Deleuze y Guatari (2004) a través del concepto de assamblage, esta capacidad de abrirse a las propiedades emergentes, fluidas y heterogéneas de la realidad, resulta el punto fuerte de la investigación lenta o slow science, también es la fuente de sus infortunios en la era de la fast science o tiempo-proyecto, es decir, bajo la presión hacia el logro de la máxima eficiencia en el menor plazo de tiempo. Y es que acoplarse a las exigencias de ese modelo de producción de conocimiento/investigación obliga a la investigación lenta a traicionar sus bases epistemológicas en un doble sentido: primero, produciendo respuestas donde debería haber preguntas; segundo, intentando crear orden y estructura, rupturas y discontinuidades en forma de representaciones métricas (ej. tablas/cuadros) y lingüísticas (ej. taxonomías) allí donde la norma es la continuidad. 
También la docencia de la investigación lenta conoce tal infortunio cada vez que el profesorado, como Sócrates con Menon, intenta que el alumnado deje atrás el ansia por la anticipación, por la búsqueda de recetas, trucos, protocolos, competencias y respuestas y en su lugar abrace tanto la incerteza, incertidumbre, complejidad de las preguntas como el pensamiento imaginativo y creativo que las envuelve (Mordechai, 2006); en definitiva, que abandonen el instrumentalismo metodológico (Mills \& Ratcliffe, 2012) y recuperen el pensamiento epistemológico como guía (Pascale, 2011, p. 4).

\section{SERENDIPIA Y MOMENTOS CONSTITUTIVOS DE UNA INVESTIGACIÓN SOCIAL CUALITATIVA}

Esta propuesta es particularmente relevante por lo que se refiere a una forma particular de investigación lenta, la investigación social cualitativa; la cual es considerada así no sólo porque su fin sea lograr una descripción densa, sino porque los dispositivos técnicos de los que se vale en el desarrollo de su trabajo de campo se basan en la observación y la escucha, es decir, en una lógica epistemológica que requiere recrearse en un tiempo lento: tiempo para observar, para entender y ponderar, para ir y volver sobre los datos y su análisis (Rivoal \& Salazar, 2013), para atender a los momentos y su continuidad.

Este tempo lento corresponde a lo que Dawn Mannay y Melannie Morgan (2015) señalan como esos otros momentos poco tenidos en cuenta pero que también son constitutivos de la investigación social cualitativa. Se trata de momentos que se perfilan en paralelo (in between) o a pesar del timing del proceso de investigación o de la aplicación de los dispositivos técnicos concretos (entrevistas, observación, etc.), distinguiendo al respecto dos tipos de momentos: "momentos previos a" y "momentos de reflexión".

Por lo que se refiere a los momentos "previos a...", aparentemente inútiles, resultan ser sin embargo fundamentales para establecer lazos, vínculos, relaciones. $Y$ no hay que olvidar que una investigación social cualitativa es predominantemente una perspectiva metodológica que depende de relaciones, las establecidas entre la persona investigadora y la persona o personas investigadas. A este respecto resulta revelador el ejemplo que la propia Dawn Mannay (2015, pp. 174-175) aporta para ilustrar este primer tipo de momentos cuando relata su experiencia en el contexto de una investigación en hogares, exponiendo los detalles del encuentro con una mujer con la que había acordado una entrevista para la investigación en curso. Aquellos minutos donde en principio todo quedaría en suspenso hasta que la entrevistada llegaba a casa (lugar establecido para el encuentro) y mientras se 
acomodaba, parecerían situarse al margen del objetivo de la investigación, y en cambio se revelaron fundamentales pues permitieron a la investigadora entender las verdaderas dimensiones materiales de la pobreza femenina.

Por otra parte, las autoras incluyen también en su tipología los "momentos de reflexión". Y este segundo tipo de momentos da cuenta de aquellos en que quienes realizan una investigación social cualitativa se toman su tiempo para intentar establecer conexiones entre los datos y la teoría creando, así, un espacio de oportunidad para una nueva forma de serendipia: la serendipia analítica. Steffen Dalsgaard y Morten Nielsen, siguiendo a Marilyn Strathern (1990), Ilaman a estos momentos momentos etnográficos y los entienden como aquellos "where the already known is transcended by establishing new associations between 'the understood... (what is analyzed at the moment of observation)' and 'the need to understand (what is observed at the moment of analysis)'. What is emphasized, however, is the volatility of the relationship established between question and answer" (Dalsgaard \& Nielsen, 2013, p. 8).

Como discurso, la serendipia analítica depende en buena medida de la sagacidad de quien investiga, lo que a mediados de los años 1980 John Lofland y Lyn Lofland Ilamaban transcending perspective (citado en Fine \& Deegan, 1996, p. 13), esto es, la capacidad para comprender que "things are not (or are not only) what they seem to be". La serendipia analítica se basa entonces en la capacidad de quien investiga para, trascendiendo los datos (o lo evidente), descender a lo profundo (incluso anómalo) de la mano de la metáfora (e incluso de la ironía), de la sutileza, la intuición, la revisión de los errores, también de la imaginación sociológica de Mills, utilizadas todas ellas como herramientas para (re)presentar la lógica de descubrimiento (que es también un régimen de temporalidad) subyacente al proceso de investigación social cualitativa en tanto que forma específica de investigación lenta. Solo así se puede poner en v-alor la lógica de discovery (o de descubrimiento) que subyace a la slow science frente a la lógica de delivery (o de entrega, producción) que subyace a la fast science.

De ahí la relevancia de prestar atención a los momentos señalados por Mannay y Morgan (2015) en tanto en cuanto implican ralentización del tiempo-proyecto, incluso ruptura de la continuidad lineal que lo define, mediante su suspensión o puesta en paréntesis. Esta suspensión es considerada no productiva desde el punto de vista de la lógica temporal del tiempo-proyecto propio de la sociedad/academia acelerada en la que cualquier 
tiempo/momento que se sitúe fuera de la forzosa sincronía del tiempo productivo es considerado un anacronismo, cuando no una aberración (Abad Miguélez, 2018).

Sin duda, desde la lógica temporal de la academia acelerada los tiempos vacíos son rechazados, negados y combatidos con movimiento, flujo, prácticas y aceleración. Sin embargo, desde la lógica del tiempo-proceso, estos momentos son claves para dar lugar a la emergencia de serendipia, de descubrimiento. Porque, en una lógica procesual del tiempo no remiten a los momentos vacíos que se cuelan entre las acciones. Así, lejos de no contar más que como ese tiempo marginal que se despliega en los márgenes muertos del trabajo de campo, se revelan como "el centro del trabajo de campo" (Palmer, Pocock \& Burton, 2018).

Desde esta perspectiva, la tipología propuesta por Mannay y Morgan (2015) resulta interesante aunque limitada, ya que no subraya convenientemente el potencial de esos momentos en los que quien realiza una investigación social cualitativa no solo apre(he)nde aspectos no sospechados del modo de vida, de ser y estar de las personas con las que trabaja, sino que aprende y adquiere conciencia de las temporalidades de los otros; quienes, al gestionar sus propios tiempos, subvierten los juegos de poder que se despliegan alrededor de la expropiación del tiempo ajeno. Porque quien hace esperar tiene poder, tiene voz, siendo así que, al rendirse ante el poder de gestión de la temporalidad propia y ajena de la cual se dotan quienes participan en una investigación social cualitativa, quien investiga deja de ser pivote, centro de gravedad o protagonista del proceso de investigación, para convertirse en sujeto de escucha, más allá de la auscultación de lo dicho con el fin de predecir, medir y comprobar (Davila \& Huici, 2018); sujeto de investigación que, por tanto, también contempla aquello que le interpela, caso del despliegue por sorpresa de la alteridad.

Por otro lado, la propuesta es interesante porque, como hemos mencionado más arriba, coloca el tiempo lento en el centro de la lógica cualitativa, lo que cualquiera que lleva a cabo una investigación advierte, desde el mismo instante en que debuta en el trabajo de campo, como algo asociado a los dispositivos técnicos de los que se vale. No en vano, más allá de los "modos tácitos" de producir conocimiento, los dispositivos técnicos de los que se vale una investigación social cualitativa (observación, entrevista, grupos, etc.) tienen como función principal la búsqueda del significado de los fenómenos, la obtención de la palabra de los sujetos de la acción social... pero todo ello enmarcado en una situación social que deseamos comprender desde el tempo lento de la reflexión como única forma posible de 
generar una descripción densa. De ahí que en todos los dispositivos técnicos el tempo y su gestión se revelen fundamentales. En este sentido, la práctica de la observación puede considerarse un espacio sin tiempo (Callejo, 2002, p. 413). Inicialmente la selección del espacio es clave, pero una vez en el espacio el tiempo se impone. En los últimos tiempos se discute sobre la extensión de ese tiempo en la comunidad. Sin quitar importancia a este debate, que en el fondo remite a la consideración del tiempo como variable o dimensión cuantitativa, más parecida a Kronos, lo que interesa destacar aquí es la lógica que conforma ese tiempo en el campo (en la comunidad). El tiempo en la comunidad está hecho de momentos, de interrupciones, de devenires (más parecido a Kairos o Airon). $Y$ otro tanto ocurre con el tempo de la entrevista abierta, aparentemente marcado por lo hablado pero en el que cobran importancia los "momentos previos a", como cuando quien entrevista aguarda, en silencio, pacientemente a que la persona con quien se entrevista encuentre su ritmo, respetando sus pausas, etc. $Y$ en cuanto al grupo de discusión, los momentos en paralelo, en suspenso, ocupan un lugar destacado en los diferentes momentos constitutivos de la sesión, conformándose en torno al silencio, a veces elusivo, necesario para pautar la resolución de temas a tratar conjuntamente. Consideraciones todas ellas que, sin embargo, se desdeñan demasiado a menudo.

\section{CONCLUSIONES}

A lo largo del texto hemos intentado exponer el alcance de describir la investigación cualitativa en tanto que investigación lenta, articulando así dos lógicas: epistemológica y temporal. Su lógica epistemológica se basa en las preguntas que lo imprevisto, lo tangencial y lo inesperado despiertan en quien investiga. Dicho de otro modo, responde a la lógica epistemológica del encuentro/descubrimiento de todo lo que no se podía prever al inicio del proceso. Por otro lado, para su despliegue se vale de una lógica temporal que se recrea en el progreso creativo del propio proceso de investigación permitiéndose dar tiempo al tiempo para observar, entender, ponderar; para, en definitiva, asumir el alcance de la capacidad performativa de la realidad. De igual manera hemos procedido al describir el tiempoproyecto como crono-lógica asociada al modo de producción de conocimiento, ciencia e investigación propia del capitalismo académico: una lógica epistemológica que se recrea en las respuestas, en la obtención y distribución de outputs directamente orientados a resolver problemas/proyectos en el corto-medio plazo. 
Esta vinculación sustancial entre lógica epistemológica y lógica temporal pasa desapercibida, o directamente se oculta e ignora, cuando la lógica de investigación (epistemológica y temporal) del capitalismo académico imperante se impone como la única opción posible desde el punto de vista de la eficiencia, la calculabilidad y la predictibilidad. $Y$ ocultar significa aquí sacrificar una epistemología en el altar de la otra imponiendo una gramática de poder, dominio y control sobre el modo de producción del conocimiento. Esto último no resulta novedoso en el seno de la tradición epistemológica occidental sustentada sobre el dominio y control de formas paralelas, alternativas, diferentes de producción de conocimiento. Lo novedoso es el pilar sobre el que se sustenta esa gramática pues, de estar sustentada sobre tropos espaciales (horizontes, campos, bordes, esferas, territorios, etc.) con el mapa como dispositivo metaforizador fundamental, pasa ahora a legitimarse desde la lógica temporal de la aceleración. Si la forma de legitimación ha cambiado, también debería hacerlo la forma en que ha de manifestarse la resistencia frente a la imposición de una forma específica de producción de conocimiento como la única posible. El futuro de la investigación social cualitativa, tal y como la conocemos, se juega en este terreno.

\section{REFERENCIAS}

Abad Miguélez, B. (2018). Regímenes de movilidad y expropiación del tiempo: la espera como cronopolítica. Arbor, 194 (788), a453. Recuperado de: https://doi.org/10.3989/arbor.2018.788n2013

Adams, V., Murphy, M. \& Clarke, A. (2009). Anticipation: Technoscience, life, affect, temporality. Subjectivity 28, 246-265. Recuperado de: https://doi.org/10.1057/sub.2009.18

Ball, S. (2003). The Teacher's Soul and the Terrors of Performativity. Journal of education policy, 18 (2), 215-228. Recuperado de: https://doi.org/10.1080/0268093022000043065

Bourdieu, P. (1992). Homo academicus. Paris: Les Éditions de Minuit.

Callejo Gallego, J. (2002). Observación, entrevista y grupo de discusión: el silencio de tres prácticas de investigación. Revista Española de Salud Pública, 76 (5), 409-422. Recuperado de: http://scielo.isciii.es/pdf/resp/v76n5/colabora3.pdf

Cannizzo, F. (2015). Academic subjectivities: Governmentality and Self-Development in Higher Education. Foucault Studies, 20. Recuperado de: https://doi.org/10.22439/fs.v0i0.4937

Dalsgaard, S. \& Nielsen, M. (2013). Time and the Field. Introduction. Social Analysis, 57 (1), 1-9. Recuperado de: https://doi.org/10.3167/sa.2013.570101

Davila Legerén, A. \& Huici Urmeneta, V. (2018). Más allá de una investigación social cualitativa extractiva: Escucha, silencio y conversación. Revista Anhanguera, 1, 119-141. Recuperado de: http://pos.anhanguera.edu.br/wp-content/uploads/2017/03/revista-anhanguera-pesquisa-quali-2018.pdf

Deleuze, G. (2017). Del bergsonismo. Buenos Aires: Editorial Cactus

Deleuze, G. \& Guattari, F. (2004). Mil mesetas Capitalismo y esquizofrenia. Pamplona: Pretextos. 
Denzin, N.K. (2013). "The Death of Data?” Cultural Studies/Critical Methodologies, 13(4), 353 -356. Recuperado de: https://doi.org/10.1177/1532708613487882

Fine, G.A. \& Deegan, J.G. (1996). Three Principles of Serendip: insight, chance and discovery in qualitative research. International Journal of Qualitative Studies in Education, 9 (4), 434-447. Recuperado de: https://doi.org/10.1080/0951839960090405

Gallas, A. (2018). Introduction: The Proliferation of Precarious Labour in Academia. Global Labour Journal, 9(1), 69-75. Recuperado de: https://doi.org/10.15173/glj.v9i1.3428

Gibbons, M., Limoges, C., Nowotny, H., Schwartzman, S., Scott, P. \& Trow, M. (1994). The New Production of Knowledge: The Dynamics of Science and Research in Contemporary Societies. London: Sage.

Keller, E. F. (1983). A Feeling for the Organism: The Life and Work of Barbara McClintock. New York: Henry Holt and Company.

Koro-Ljungberg, M. \& Barko, T. (2012). 'Answers,' Assemblages, and Qualitative Research. Qualitative Inquiry, 18 (3), 256-265. Recuperado de: https://doi.org/10.1177/1077800411431562

Levy, D. M. (2007). No time to think: Reflections on information technology and contemplative scholarship. Ethics and Information Technology, 9, 237-249. Recuperado de: http://faculty.washington.edu/dmlevy/Levy_No_Time_to_Think.pdf

Lofland, J. \& Lofland, L. (1984). Analyzing Social Settings: A Guide to Qualitative Observation and Analysis. Belmont (CA): Wadsworth Publishing Co Inc.

MacLure, M. (2013). The Wonder of Data. Cultural Studies/Critical Methodologies, 13 (4), 228-232. Recuperado de: https://doi.org/10.1177/1532708613487863

Mannay, D. \& Morgan, M. (2015). Doing ethnography or applying a qualitative technique? Reflections from the "waiting field". Qualitative Research, 15 (2), 166-182. Recuperado de: https://journals.sagepub.com/doi/10.1177/1468794113517391

Mills, D. \& Ratcliffe, R. (2012). After Method? Ethnography in the Knowledge Economy. Qualitative Research, 12, 147-64. Recuperado de: https://doi.org/10.1177/1468794111420902

Mordechai, G. (2006). Welcoming Confusion, Embracing Uncertainty: Educating Teacher Candidates in an Age of Certitude. Paideusis, 15 (2), 15-25. Recuperado de:

http://journals.sfu.ca/pie/index.php/pie/article/view/74/22

Morrish, L. \& Sauntson, H. (2016). Performance Management and the Stifling of Academic Freedom and Knowledge Production. Journal of Historical Sociology, 29(1), 42-64. Recuperado de: https://doi.org/10.1111/johs.12122

O'Neill, M., Martell, L., Mendick, H. \& Müller, R. (eds.) (2014). Slow Movement/Slow University: Critical Engagements. Introduction to the Thematic Section. Forum Qualitative Sozialforschung / Forum: Qualitative Social Research, 15 (3). Art. 16. Recuperado de: http://dx.doi.org/10.17169/fqs-15.3.2229

Pascale. C.M. (2011). Cartographies of Knowledge- Exploring Qualitative Epistemologies. Thousand Oaks, California: Sage.

Pels, D. (2003). Unhastening Science. Autonomy and Reflexivity in the Social theory of Knowledge. Liverpool, UK: Liverpool University Press.

Rivoal, I. \& Salazar, N.B. (2013). Contemporary Ethnographic Practice and the Value of Serendipity. Social Anthropology, 21(2), 178-185. Recuperado de: https://doi.org/10.1111/1469-8676.12026 
Ryan-Flood, R., \& Gill, R. (2010). Breaking the silence: The hidden injuries of neo-liberal academia. En R. RyanFlood, \& R. Gill (Eds.), Secrecy and silence in the research process: feminist reflections (pp. 228-244). Abingdon, Oxon; New York, NY: Routledge.

Schwandt, T. A. (2008). Educating for intelligent belief in evaluation. American Journal of Evaluation, 29(2), 139150. Recuperado de: https://journals.sagepub.com/doi/10.1177/1098214008316889

Slaughter, S. \& Leslie, L. (1997). Academic Capitalism. Politics, Policies and the Entrepreneurial University. Baltimore, Maryland (USA): Johns Hopkins UP.

Smith, S. (2015). Multiple temporalities of knowing in academic research. Social Science Information, 54 (2), 149176. Recuperado de: https://doi.org/10.1177/0539018414566421

Strathern, M. (1990). Artefacts of History: Events and the Interpretation of Images. En J. Siikala (Ed.), Culture and History in the Pacific (pp. 25-44). Helsinki: Suomen Antropologinen Seura.

Torrance, H. (2008). Building Confidence in Qualitative Research Engaging the Demands of Policy. Qualitative Inquiry, 14 (4), 507-527. Recuperado de: https://doi.org/10.1177/1077800407309380

Vostal, F. (2015). Academic life in the fast lane: The experience of time and speed in British academia. Time \& Society, 24 (1), 71-95. Recuperado de: https://doi.org/10.1177/0961463X13517537

Ylijoki, O-H. (2016). Projectification and conflicting temporalities in academic knowledge production. Theory of Science, 38 (1), 7-26. Recuperado de: http://teorievedy.flu.cas.cz/index.php/tv/article/view/331/363 